\title{
La « malédiction » d'Apulée : une interprétation pragmatique et rhétorique
}

The 'Curse' of Apuleius: a Pragmatic and Rhetorical Interpretation

\section{Benoît Sans}

\section{(2) OpenEdition}

\section{Journals}

Electronic version

URL: https://journals.openedition.org/aad/1851

DOI: 10.4000/aad.1851

ISSN: 1565-8961

Publisher

Université de Tel-Aviv

\section{Electronic reference}

Benoît Sans, "La « malédiction » d'Apulée : une interprétation pragmatique et rhétorique",

Argumentation et Analyse du Discours [Online], 13 | 2014, Online since 14 October 2014, connection on

21 September 2021. URL: http://journals.openedition.org/aad/1851 ; DOI: https://doi.org/10.4000/aad. 1851

This text was automatically generated on 21 September 2021.

\section{c) (†) $९$}

Argumentation \& analyse du discours est mis à disposition selon les termes de la licence Creative Commons Attribution - Pas d'Utilisation Commerciale - Pas de Modification 4.0 International. 


\title{
La « malédiction » d'Apulée : une interprétation pragmatique et rhétorique
}

The 'Curse' of Apuleius: a Pragmatic and Rhetorical Interpretation

\author{
Benoît Sans
}

\section{La malédiction d'Apulée}

1 Sabratha, vers 159 de notre ère ${ }^{1}$. Apulée doit se défendre d'une accusation de magie devant un tribunal présidé par le proconsul d'Afrique Claudius Maximus. Il avait récemment épousé Pudentilla, la mère de son ami Sicinius Pontianus, à la demande ce dernier, mais il est à présent soupçonné d'avoir ensorcelé sa future femme pour qu'elle accepte le mariage contre son gré. L'accusateur officiel n'est autre que Sicinius Pudens, le plus jeune fils de Pudentilla et son premier mari, mais le véritable instigateur du procès, contre lequel est tourné le discours que nous avons conservé, est Sicinius Aemilianus, le frère du mari défunt ${ }^{2}$. Il a vu d'un mauvais œil l'arrivée d'un nouveau venu dans la famille: il semble que le véritable enjeu de cette lutte soit le riche patrimoine de Pudentilla (Schenk 2002: 43-46; Walker 2000: 122-123; Hunink 2001 : 13 ; 1997 : 12-18; Graf 1994: 81-83; Fick 1992: 29-46). Graf a proposé pour le procès d'Apulée une interprétation socio-anthropologique, inspirée des théories de Marcel Mauss, devenue classique (Graf 1994: 81-83; 104-105; Mauss1973: 1-141): le philosophe appartient à l'élite intellectuelle itinérante de l'époque impériale, proche des milieux du pouvoir, et apparaît comme un élément hétérogène qui vient bouleverser les structures établies dans la petite communauté fermée d'Oea. Ses attitudes curieuses et son intérêt pour certaines matières, son ascension sociale fulgurante, son succès ${ }^{3}$, n'ont pu manquer d'attirer la malveillance et le soupçon. La communauté, se sentant menacée dans ses fondements, réagit par une accusation de magie afin de résoudre les tensions et les rivalités qui se sont créées. Le sorcier est 
toujours une figure marginale, et toute personne dans cette situation est un sorcier potentiel ${ }^{4}$.

Les mauvais sorts et les pratiques magiques sont punissables 5 . La stratégie de défense repose essentiellement sur le status definitionis (Hijmans $1994: 1761)$ : Apulée ne nie pas les faits qui lui sont reprochés, mais récuse leur qualification. L'auteur clame qu'il est philosophe et non magicien, et son procès devient celui de la philosophie ${ }^{6}$. Dans une longue introduction, mais aussi dans la suite du discours, il soigne son image en se servant de sa culture hellénique, de ses connaissances encyclopédiques, du platonisme et de sa curiosité scientifique tout en fustigeant son adversaire, Aemilianus, qu'il dépeint comme un paysan rustre et ignorant. Avec assurance et souci du détail, Apulée repousse successivement les attaques quant à son mode de vie puis celles qui concernent des actions isolées assimilées à des actes magiques, avant d'en venir, dans une troisième partie, à l'accusation principale portant sur son mariage avec Pudentilla ${ }^{7}$. L'un des faits troublants évoqués dans la deuxième partie de ces réfutations est l'adoration d'une statuette : l'accusateur reproche à Apulée le secret de sa fabrication, le choix du matériau, la forme de sceletus et le nom «basileus» (« roi ») dont Apulée se sert pour l'appeler. Tous ces indices laissent penser que l'accusé entretient commerce avec des puissances occultes. L'orateur réfute ces accusations qu'il qualifie de mendacia (mensonges): tout le monde pouvait connaître l'existence de cette statuette, commandée chez un artiste connu et réputé (Cornelius Saturninus), cité devant le tribunal de Maximus ; la matière n'a fait l'objet d'aucune recherche particulière, le bois provient d'un cadeau de Pontianus ; quant à la forme de la statuette, Apulée répond en montrant qu'il s'agit d'une représentation du dieu Mercure, auquel il affirme vouer un culte quotidien. Il considère alors l'accusation de son adversaire comme un sacrilège et s'indigne. Cette déformation du propos n'a rien de surprenant au regard de l'explication de Graf et aurait déjà pu figurer dans le discours de la partie adverse : à l'époque d'Apulée, la séparation théorique entre la magie d'une part, la religion, la science et la philosophie de l'autre, est depuis longtemps consommée ${ }^{9}$; le magicien a été mis au banc de la société et du culte traditionnel, il peut être poursuivi pour ses pratiques considérées comme criminelles.

3 Mais dans le passage qui suit immédiatement la réfutation quant à la forme de la figurine, Apulée se retourne brutalement contre son adversaire d'une manière qui semble incompatible avec la ligne de défense :

At tibi, Aemiliane, pro isto mendacio duit deus iste superum et inferum commeator utrorumque deorum malam gratiam semperque obuias species mortuorum, quidquid umbrarum est usquam, quidquid lemurum, quidquid manium, quidquid laruarum, oculis tuis oggerat, omnia noctium occursacula, omnia bustorum formidamina, omnia sepulchrorum terriculamenta, a quibus tamen aeuo et merito haud longe abes. (Apol. 64, 1) ${ }^{10}$.

Mais toi, Aemilianus, pour prix de ton mensonge, puisse ce dieu qui est l'intermédiaire entre les mondes supérieur et inférieur t'apporter la malveillance des divinités de l'un et de l'autre, mettre toujours sur ta route les fantômes des morts et assembler sous tes yeux tout ce qu'il peut y avoir d'ombres, de lémures, de mânes, de larves, toutes les frayeurs de la nuit, toutes les horreurs des bûchers, les terreurs des tombeaux, dont tu n'es cependant pas si éloigné par l'âge et le caractère.

L'orateur lance ici une imprécationà l'encontre de son adversaire et trahit ainsi sa connaissance des arts obscurs ${ }^{11}$. Il semble commettre une imprudence qui pourrait soulever le doute ${ }^{12}$. On a donc tout naturellement tenté d'expliquer cette 
inconséquence de diverses façons et en ayant recours à différentes disciplines. Or, si le discours d'Apulée est un texte remarquable tant par sa valeur historique que littéraire, ces deux aspects ne doivent pas faire oublier qu'en tant que discours qui vise à persuader, il relève également de la rhétorique : la technique rhétorique elle-même, décrite dans les traités antiques et toujours étudiée aujourd'hui, peut être, au même titre que la philologie ou l'histoire, un outil pour la compréhension du texte et l'élucidation de passages problématiques. Les enseignements que l'on pourrait alors retirer de la pratique, en appliquant les concepts à un discours concret, en reconstituant la stratégie et les choix de l'orateur, pourraient toujours inspirer le praticien moderne de la rhétorique ${ }^{13}$.

5 Avant de nous pencher sur les solutions qui ont été proposées, puis de montrer par une critique interne du passage et une analyse de l'argumentation qu'Apulée n'a pas ici commis de maladresse, il nous faut préciser au mieux le sens de cet extrait.

\subsection{Une defixio?}

6 Tout d'abord, il ne sera pas inutile de nous demander ce qui, dans le passage précité, évoque une pratique magique, et si l'extrait peut être effectivement compris comme tel. Plusieurs indices, comme le contexte de crise, et plus particulièrement, celui d'une action judiciaire, la présence d'Hermès en fonction de psychopompe et de messager, l'énumération des horreurs peuplant les Enfers et surtout des âmes des défunts, le style très soigné, les mots rares ou étranges (lemurum, manium, laruarum, oggerat, occursacula, formidamina, terriculamenta) et l'optatif archaïque duit ${ }^{14}$ qui rappellent une incantation codifiée, nous mettent sur la piste d'une forme de malédiction très répandue dans le monde gréco-romain, bien attestée par la littérature et les témoignages archéologiques : la defixio (gr. katadesmos) ${ }^{15}$.

7 Ce rituel magique consistait à " lier » ou contraindre une personne en la vouant à des divinités infernales ou à un intermédiaire : on soumettait ainsi l'autre à son pouvoir pour le rendre incapable d'agir selon sa propre volonté. Le plus souvent, la malédiction et le nom de la personne visée étaient inscrits sur des lamelles en matériau dur, comme le plomb, qui étaient ensuite cachées dans une sépulture ou dans le sanctuaire d'une divinité. Ce rite pouvait éventuellement être exécuté avec l'aide d'un professionnel (Graf 1994 : 170-171). Mais si les procès de l'Antiquité donnaient souvent lieu à une telle pratique (Faraone 1999 : 99-121), il convient d'analyser le rapprochement entre celle-ci et le discours d'Apulée avec davantage de circonspection, car il serait particulièrement mal venu dans la défense de l'accusé de lancer contre son adversaire une malédiction identifiable et assimilable à un type précis ou connu, et parce que d'autres éléments invitent, selon moi, à rejeter cette hypothèse.

Le moment, tout d'abord, ne convient pas : la defixio aurait dû avoir lieu avant le procès pour protéger l'accusé, empêcher l'accusation de parler ou rendre son intervention hésitante ; ou bien encore après le procès, en représailles. Cette première remarque est d'ailleurs également valable pour d'autres sortes de malédictions. Dans le cas présent, la partie adverse a déjà eu l'occasion de s'exprimer et d'exposer en long et en large des griefs en apparence accablants. L'hypothétique vengeance qui s'abattra sur Aemilianus dépend du dieu, offensé par le mensonge, et n'est pas fixée dans le temps : elle pourra très bien s'exercer à l'issue du procès en cours et n'avoir aucune influence particulière sur le sort d'Apulée. Ensuite, si la defixio semble être un acte relativement banal et peut 
être accomplie dans des lieux publics, elle n'en reste pas moins un acte privé et secret (Faraone 1991: 17). Or le discours d'Apulée désigne clairement l'auteur et la victime. Enfin, la formulation de la malédiction ne correspond pas à ce que l'on trouve habituellement dans les messages de defixio. Citons ici A. Audollent (1903 : 37-38), qui rappelle le caractère double de la contrainte exercée par l'envoûtement :

Ceux qui les rédigeaient se sont toujours proposés, au moyen d'expressions à l'efficacité desquelles ils ajoutaient la foi la plus entière, d'une part d'immobiliser, et pour me servir du mot technique de fixer une personne, de telle sorte qu'elle ne puisse échapper aux châtiments qu'ils lui souhaitaient; d'autre part d'obliger les dieux à intervenir en leur faveur.

Dans l'extrait qui nous concerne, l'orateur n'entend exercer aucune contrainte sur le dieu qu'il invoque, il l'invite simplement à tirer vengeance de l'offense qui a été faite sur l'un de ses fidèles dévoués mais aussi, à titre individuel, sur sa propre personne divine. Les defixiones que nous avons conservées sont le plus souvent exprimées avec un verbe de type déclaratif à la première personne de l'indicatif présent ou se donnent comme une injonction à l'impératif, au subjonctif ou encore à l'indicatif futur (Graf 1994 : 143-147). Elles se caractérisent donc par une force illocutoire ${ }^{16}$ très forte, qui traduit un profond désir que l'action visée se réalise. Chez Apulée, on trouve le subjonctif oggerat, mais celui-ci est juxtaposé à l'optatif duit qui ouvre l'adresse. Ce premier verbe précise et diminue à la fois considérablement la force (illocutoire) de la demande qui est faite au dieu; l'intensité du désir et l'engagement de l'énonciateur sont affaiblis. Les paroles de l'orateur relèvent donc plutôt du souhait malveillant, de la prière ou de l'imprécation spontanée ${ }^{17}$.

\section{2. Une audacieuse parodie?}

10 D'autres interprétations sont en lien étroit avec la manière dont l'œuvre est envisagée dans son ensemble. D'après les chercheurs modernes, à l'exception notable de Winter $(1969$; 1968), selon qui la publication du discours serait le fait de sténographes zélés, il est peu probable que le texte que nous avons conservé soit celui qui fut prononcé lors du procès. Comme c'est le cas pour les discours de Cicéron, le plaidoyer d'Apulée aurait subi a posteriori des remaniements plus ou moins importants ${ }^{18}$. Divers arguments ${ }^{19}$ comme la longueur du discours, le peu de temps dont disposait Apulée pour préparer sa défense, les nombreuses allusions et citations d'œuvres littéraires ou scientifiques, l'agressivité du ton et la violence des attaques personnelles, y compris celle dont il est ici question, ont été avancés sans qu'aucun d'entre eux ne paraisse décisif ${ }^{20}$. Certains éléments (écoulement du temps, production de preuves, lectures de documents, déictiques) font référence à une situation extra-linguistique (Hijmans 1994 : 1719), mais ont pu être insérés pour créer un effet de réel. À défaut de certitude, l'hypothèse la plus largement acceptée est que le discours qui nous est parvenu renvoie à un procès réel, mais que le texte aurait été revu ultérieurement dans une mesure qu'il est difficile d'apprécier ${ }^{21}$.

11 D'autres préfèrent voir l'Apologie comme une habile et divertissante fiction (Schneider 2009 : 395 ; Hunink 1997 : 26 ; Sallmann 1995 : 137-158 ; McCreight 1991 : 29-41 ; Stock 1985 : 353-386) mise sous la forme d'un procès judiciaire et comme une véritable démonstration d'art oratoire et d'érudition, proche des exercices de rhétorique (progymnasmata) et de la déclamation ${ }^{22}$ (controverses et suasoires), et s'insérant dans le cadre de la seconde sophistique ${ }^{23}$. Pour Gaide (1993 : 230) ou encore McCreight (1991: 
255-256), le passage examiné ici ne serait qu'une fausse malédiction à vocation purement parodique et simplement destinée à amuser le public. La longue énumération de créatures infernales s'achève d'ailleurs par un sarcasme où Apulée compare son adversaire à ses futurs tourmenteurs. Il est vrai que la tactique d'Apulée consiste le plus souvent, après avoir dénoncé l'erreur d'interprétation de ses adversaires, à tourner l'accusation en ridicule en la poussant jusqu'à l'absurde (Apol. 9, 3;30, 1 ; 32;41, 1 ; voir également Sandy 1997 : 141) et à se moquer des plaignants et de leur ignorance jusqu'à la provocation ${ }^{24}$. Toutefois, de telles manœuvres, insultes ou railleries ad personam, visant à ternir l'image de l'adversaire, étaient tout à fait courantes dans les tribunaux antiques et pouvaient faire partie de la technique ${ }^{25}$. À l'exception de la chute, aucun élément comique ne transparaît dans l'imprécation elle-même (Hunink 1997: 169), dont le style très rythmé est au contraire empreint de gravité. La pique finale ne retire rien aux précédents propos d'Apulée et fait seulement office de mise à distance, qui facilite le passage vers un nouveau mouvement de pensée, l'opposition entre Aemilianus, proche de la famille des monstres, et la Platonica familia à laquelle appartient Apulée.

Ne s'écartant que très peu de l'hypothèse comique, Hertz rappelle qu'Apulée et ses contemporains croyaient en l'existence de la magie et en la puissance des dieux (Hertz 2010 : 116 ; Habermehl 2002 : 290) : l'affaire est prise au sérieux et pour le défendeur, il n'y a pas matière à plaisanter exagérément. Toutefois, cette chercheuse voit dans ce passage une nouvelle preuve de désinvolture, ainsi que du talent tactique et littéraire d'Apulée (Hertz 2010 : 116-117), qui n'hésite pas à jouer avec le soupçon qui pèse sur lui ${ }^{26}$. Au début du chapitre 64, l'orateur repousse les limites de l'audace et du style : il joue un rôle ${ }^{27}$ (Habermehl 2002 : 289 ; Harrison 2000 : 74-75) et imite un méchant magus, précisément pour montrer qu'il n'en est pas un. Pour Hertz, c'est en somme une forme élaborée d'argument de Corax, tel qu'il a été décrit par Aristote ${ }^{28}$, qui est à l'œuvre dans l'extrait cité. Néanmoins, cette tactique rhétorique, si intelligente et séduisante soitelle, est largement implicite et n'est appréhensible qu'au second degré. Elle apparaît risquée et peu rentable : risquée parce qu'elle implique la reconnaissance du caractère magique de l'imprécation; peu rentable, car, selon Hertz (2010: 118), sa cible privilégiée est sans doute le proconsul Claudius Maximus, qui est présenté par Apulée comme un homme instruit et versé en philosophie; or, dans les chapitres 63-65, l'orateur semble aussi faire appel à un plus large public que le juge et les conseillers qui l'entourent (63, 5: auditisne reclamationem omnium qui adsunt?; 65, 1: quosdam circumstantium ; 65, 8 : ut omnium assensus declaravit), et cela est d'autant plus vrai si l'on estime qu'Apulée a pu envisager la publication de son discours et une diffusion plus vaste encore.

De façon plus générale, dans l'hypothèse d'une déclamation ou d'un remaniement, on pourrait également penser que la rupture avec la réalité aurait conduit Apulée à laisser libre cours à ses émotions et à sa fantaisie. Cependant, quel que soit le cas de figure dans lequel on se trouve, les données du problème ne sont guère modifiées. Pour celui qui prétend au titre d'orateur, même pour le temps d'une ingénieuse fiction, la contradiction interne constitue une erreur fatale qui ne serait pas sans conséquences pour la bonne réception du discours auprès d'un large public, plus ou moins expert. Les discussions sur l'authenticité du discours montrent que l'auteur est au moins parvenu à maintenir l'apparence de la réalité, ce qui constitue sans doute un des sommets de l'art (Schneider 2009 : 412-413 ; Sandy 1997 : 131-132). Qu'Apulée ait effectivement prononcé ce plaidoyer, tel que nous le lisons aujourd'hui, lors de son procès, ou qu'il ait revu sa 
copie en vue de sa diffusion, il est impensable que le spécialiste de rhétorique qu'il était ait prêté le flanc à la critique en commettant une telle maladresse. En outre, il renouvelle son imprécation de manière succincte en 75,1 , dans un passage où il s'emploie à dresser un portrait peu reluisant de son adversaire.

Il me paraît donc préférable de chercher une explication rhétorique plus immédiate en interrogeant le cotexte, en reprenant l'enchaînement des idées et des arguments, afin de tenter de comprendre comment le passage discuté peut participer à la persuasion. Je me servirai pour cela de la théorie aristotélicienne des preuves techniques (ethos, pathos, logos); celle-ci, qui avait été transmise aux Latins, était toujours discutée, adaptée et enseignée aux premiers siècles de l'Empire par des sophistes et rhéteurs comme Apulée ${ }^{29}$, et reste un outil d'analyse pertinent aujourd'hui.

\section{Analyse rhétorique et pragmatique}

\section{1. Le logos}

En ce qui concerne le logos tout d'abord, au chapitre 63, la réfutation d'Apulée s'inscrit parfaitement dans la stratégie globale que j'ai rappelée en tête de cet article : l'auteur ne nie pas être en possession de la statuette, mais récuse l'identification et l'utilisation que lui prête l'adversaire. Apulée s'emploie donc à dénoncer le nouveau mensonge (mendacium) de son accusateur et à rétablir la vérité par une rupture de liaison ${ }^{30}$. Il s'appuie pour cela sur l'évidence de la preuve extra-technique qu'il s'empresse de produire devant ses juges (cedo eum...considerent) et qu'il appuie par un récit sur son origine et par une ekphrasis (Schneider 2009 : 409 ; Schenk 2002 : 35 ; Harrison 2000 : 74). Après avoir établi la véritable nature de la représentation, l'orateur se lance dans une série de questions rhétoriques sur un ton indigné et prend son public à témoin. Progressivement, par une suite de définitions, Apulée opère un glissement rhétorique qui requalifie l'action d'Aemilianus ainsi que l'effigie. L'expression mendacii uestri damnationem appelle à la condamnation de l'adversaire, le terme calumniam implique une accusation fausse et injurieuse. L'objet est quant à lui présenté comme sollemne et commune simulacrum et cette qualification est opposée à magicum; de manière flatteuse, le plaideur affirme que les pieuses mains du proconsul (tam puris et tam piis manibus) sont dignes de toucher la représentation du dieu. Le philosophe tend donc à considérer le propos de son adversaire comme une accusation d'impiété ou de sacrilège, par laquelle il tente de susciter l'émoi et qu'il cherche aussitôt à repousser.

L'ekphrasis des paragraphes 63, 7 à 9, livrée sous forme d'exclamations successives, pleine d'éloges et d'expressions subjectives n'est pas un argument à proprement parler, mais acquiert une double fonction rhétorique par ses implications ${ }^{31}:$ identifier les traits de la statuette avec ceux du dieu Mercure en la rendant présente aux yeux du public et faire voir également la vénération d'Apulée pour cette divinitée ${ }^{32}$. L'orateur admire jusqu'à la forme du dieu, la beauté de sa représentation (ut decenter...quam lepide...quam... festive) et de ce fait, témoigne son affection, et tourne toujours un peu plus en dérision la confusion commise par Aemilianus. L'ensemble du chapitre est très vivant: les accumulations, exclamations et déictiques accompagnent l'animation de l'orateur et font allusion à son actio.

17 Après l'habile ekphrasis, qui écarte le soupçon sur la nature de la statuette et canalise l'attention du public, le dernier paragraphe marque un changement de direction dans 
l'argumentation d'Apulée : c'est désormais Aemilianus qui devient l'objet du soupçon. Apulée tente de fournir une explication à la (soi-disant) méprise de son adversaire dans une "alternative plombée» (Whitehead 1979: 474-495) : même s'il évoque deux possibilités, celles-ci confinent au même but, et la dernière proposition privilégie la seconde hypothèse en énonçant la règle qui doit être appliquée : hunc denique qui laruam putat, ipse est laruans ${ }^{33}$. L'orateur emploie ici une sorte d'argument ad hominem ou de rétorsion (Eggs 1994 : 92-96 ; Perelman \& Olbrechts-Tyteca 2008 [1958] : 148-153 ; 274), procédé par lequel il tente de rejeter le discrédit sur son adversaire en retournant contre lui sa propre accusation ${ }^{34}$ et qu'il renforce par un jeu de mots (laruam / laruans). Cette manœuvre rhétorique est précisément celle qui annonce et autorise l'imprécation qui suit, mais qui ne constitue pourtant pas un argument en elle-même: puisque l'adversaire est incapable de peser le poids de ses paroles, le dieu n'a qu'à mettre des laruae sur sa route. L'indignation est mêlée d'humour et l'agression se termine par le sarcasme ("d'ailleurs, tu ressembles déjà aux laruae »). Dans la suite du chapitre 64, Apulée affirme qu'à travers la statuette de Mercure, il vénère le Dieu-roi platonicien, origine de toute chose $^{35}$. En revendiquant son appartenance au platonisme, en rappelant le Phèdre de Platon, qui n'exclut nullement l'existence des dieux, Apulée montre non seulement son humble respect face au divin, mais prétend aussi que les philosophes atteignent une compréhension supérieure du monde et de la divinité. Il ne faut pas chercher bien loin pour savoir où l'auteur du Démon de Socrate a pu trouver le modèle d'une telle défense ${ }^{36}$. À la fin du chapitre $(64,8)$, ce dernier joue à nouveau avec le soupçon de magie en refusant de nommer l'indicible ${ }^{37}$.

\section{2. Le pathos}

18 Au niveau des émotions, Apulée exprime d'abord, dans le chapitre 63, son indignation et cherche à la communiquer à l'auditoire. L'émotion est à la fois dite implicitement par les termes négatifs qui qualifient l'action de l'adversaire et la réaction qu'elle appelle, montrée par la série insistante des questions rhétoriques, et suscitée auprès du public ${ }^{38}$ : Apulée représente une scène dans laquelle un agent déterminé, l'accusateur, commet une injustice contre une ou plusieurs victimes clairement identifiées et qui ne l'ont pas mérités9. Il est important de souligner ici un glissement obtenu par le biais du pathos: l'acte blâmable ne semble plus offenser le défendeur, mais la personne même du dieu, qui a été confondu avec un vulgaire sceletus. Apulée témoigne aussi de son admiration pour Mercure avec des modalités semblables (qualifications subjectives, exclamations en série graduelle). Quant à l'imprécation elle-même, elle appelle le malheur et la colère du dieu sur l'interlocuteur, et tente donc de susciter chez lui une appréhension liée aux conséquences indésirables de ses actes ${ }^{40}$, ou de ses accusations inconsidérées. Comme l'avaient noté Abt ou Habermehl ${ }^{41}$, l'émotion visée est la peur.

Dans ce dispositif, la moquerie qui clôt l'énumération des horreurs du monde d'en bas perd son côté humoristique si l'on se place du point de vue de l'adversaire. Celui-ci ne peut que rire jaune : avec merito, Apulée rappelle à Aemilianus qu'en raison de son attitude, il sera sans doute amené à faire la connaissance d'êtres au caractère aussi peu amène que lui ; avec aeuo, il prédit qu'en raison de son âge avancé, cette rencontre se rapproche à grands pas; l'adverbe d'opposition tamen laisse d'ailleurs penser que l'intervention de Mercure sera bientôt inutile. Autrement dit, Apulée accentue le sentiment en augmentant, sur le plan qualitatif, la gravité des conséquences à redouter et, sur l'axe temporel, la proximité et le degré de probabilité de celles-ci ${ }^{42}$. Mais en 
cherchant à éveiller une certaine appréhension chez son accusateur, Apulée témoigne aussi de son propre respect mêlé de crainte face au dieu, ainsi que de celui de ses auditeurs. Ceci nous conduit à la dernière preuve technique.

\section{3. L'ethos}

20 C'est au niveau de l'image que l'orateur donne de lui-même à travers son discours, que la stratégie d'Apulée révèle, à mon sens, tout son intérêt. Cette preuve occupe déjà une place très importante chez Aristote (Rhét. I, 2, 1356a) et elle a été particulièrement mise en valeur par les rhéteurs romains (Amossy 2006 ${ }^{2}$ : 70-71; voir aussi note 50) ou, de façon générale, par la société antique. C'est dans cette perspective qu'il faut reprendre l'interprétation anthropologique de Graf, que j'ai résumée au début de cet article, et selon laquelle le sorcier est, déjà dans la société de l'Antiquité tardive, une figure marginalisée. Le comportement d'Apulée, ses recherches scientifiques et philosophiques, son désir d'approcher de trop près ce qui est du domaine du sacré ont pu paraître étranges aux yeux des indigènes et être perçus comme des pratiques magiques dans la culture populaire de personnes méfiantes et non cultivées. Dès lors, selon Habermehl (2002: 291), le principal souci d'Apulée, accusé d'être un sorcier, est un désir de reconnaissance. L'orateur insiste en effet à plusieurs reprises sur le fait qu'il est connu et apprécié de tous (Apol. 55, $10 ; 73,94)$. De là aussi, sans doute, la transformation de ce procès en celui de la philosophie, de la médecine, de la curiosité scientifique ou de l'éloquence. En étalant ses connaissances et en faisant la démonstration de sa maîtrise, Apulée affirme son statut social et son appartenance à une élite qui entretient une culture reposant sur une éducation dépourvue de visée pratique, inutile, et donc inoffensive et respectable ${ }^{43}$ (Riess 2008: 58-59; Rives 2008 : 35-49). Dans les deux chapitres étudiés, Apulée tente de faire entrer sa pratique religieuse et son rapport au divin dans le cadre de la normalité, et il veut susciter l'émotion autour de valeurs partagées, comme le respect ou l'amour du dieu ${ }^{44}$.

Dans le même temps, le caractère polémique et fortement polarisé du discours judiciaire donne à la preuve éthique une importance accrue: elle devient à la fois l'objet et l'enjeu de la lutte (Albert \& Nicolas 2010:42); à l'ethos correspond un antiethos, portrait discursif de l'adversaire qui, comme son vis-à-vis, se doit d'être crédible et soigné afin d'induire une persuasion par la négative. Cet aspect particulier du discours d'Apulée et l'originalité dont il fait preuve ont été bien montrés par Hertz dans l'article précédemment cité (Hertz 2010), ainsi que par Diouf (2005 : 92-99). Il permet également de dépasser le sens strict des mots et de mieux comprendre la stratégie globale d'Apulée autour du principe du tiers-exclu : il n'y a que deux voies possibles, être dans ou en dehors de la communauté ; le sorcier en est exclu et ne respecte pas les dieux qu'il manipule; dès lors, s'il honore les dieux de la cité, Apulée ne peut être un magicien. Dans l'extrait concerné ici, après avoir soigné son ethos en admirant l'effigie du dieu, Apulée s'en prend directement à l'image de son accusateur, jusque dans ses traits physiques : l'antagonisme culmine dans une agression particulièrement violente, sous la forme d'une imprécation. L'adversaire est brutalement expulsé dans le domaine du sacrilège. Après l'imprécation, l'opposition est relayée par le connecteur ceterum ${ }^{45}$ et les embrayeurs (At tibi / Ceterum Platonica familia) : ceux-ci isolent davantage Aemilianus et assurent la transition avec la suite du chapitre 64, où l'orateur continue d'asseoir sa religiosité et veut donner à son platonisme l'aspect rassurant de la conformité. 


\section{4. Acte et figure de l'imprécation} comme dans d'autres (Apol. 55-56), la volonté d'Apulée de se présenter comme un philosophe dévot qui honore les dieux, croit en leur existence, en leur pouvoir sur le monde et en leur nature supérieure, sans chercher à entretenir commerce avec eux d'égal à égal (Harrison 2008 : 9 ; 2000 : 50 ; Schenk 2002 : 35-36 ; Sandy 1997 : 144 ; 146 ; Fick 1991 : 14-31; Regen 1971 : 94). Mais le lien avec l'imprécation qui ouvre le chapitre 64 n'a jamais été fait, sinon dans l'idée qu'elle pourrait desservir l'orateur (Hertz 2010 : 113). La pragmatique, et plus précisément la théorie des actes de langage, permet d'apporter une réponse différente. Les philosophes du langage, comme Austin (1962), Searle (1982) ou encore Vanderveken (1988), nous apprennent que le succès des actes illocutoires, c'est-à-dire des actes accomplis par le langage, dépend de certains paramètres ou nécessite certaines conditions préalables ${ }^{46}$. Ainsi, des actes déclaratifs comme « je vous marie », « je vous bénis » ou « je vous condamne », exigent, pour leur éventuelle application, l'autorité ou les pouvoirs conférés par une institution extralinguistique. Dans le cas présent, en dévoilant le prétendu mensonge d'Aemilianus et en montrant sa propre dévotion pour le dieu Mercure, Apulée se dote de l'autorité suffisante pour en appeler à la colère divine et exprimer ainsi un désir de vengeance. L'imprécation affirme et confirme en même temps cette qualité car elle nécessite que son auteur reconnaisse la puissance de la divinité invoquée et sa capacité à intervenir sur le monde, dans le présent ou le futur. Autrement dit, par son énonciation même, Apulée adopte la posture du fidèle outré qui s'oppose radicalement à celle du magicien : il craint le courroux du dieu, l'appelle à se faire lui-même justice pour un sacrilège, mais ne peut en aucun cas le contraindre à agir pour servir des buts personnels. En allant plus loin encore, on peut même dire que les conditions nécessaires à la réalisation de l'imprécation sont présupposées ${ }^{47}$ dans l'énonciation ${ }^{48}$, ce qui rend l'attaque particulièrement difficile à contrer. Le génie d'Apulée est d'avoir exploité, dans des circonstances très particulières, mais tout à fait appropriées, les propriétés d'un acte de langage à des fins rhétoriques et persuasives, afin de construire son ethos.

D'autres éléments dans le contenu de l'invocation vont également dans ce sens : la divinité principale jouit d'un culte largement répandu et reconnu, la fonction qui lui est assignée fait partie de ses attributions habituelles et la terrifiante vision offerte du monde d'en bas ne s'écarte guère de la tradition. Quant aux Lémures, Mânes et Larves, si l'on en croit un extrait du Démon de Socrate ${ }^{49}$, ils renvoient au culte des ancêtres dont on connaît l'importance dans la vie quotidienne des Romains ${ }^{50}$ : les invoquer est donc un moyen particulièrement efficace de faire pression sur l'adversaire, mais aussi de montrer sa propre intégrité. En résumé, le procédé utilisé par Apulée pour exploiter l'erreur de son accusateur est à la fois simple, immédiat et redoutable, mais il n'a rien de magique : l'imprécation dans son ensemble, par les conditions que présuppose sa réalisation et par son contenu étudié, confère à son auteur la légitimité du sacré et, par effet de miroir, rejette le sacrilège sur l'adversaire.

Certes, malgré l'ampleur et la variété de ses connaissances, on ne peut soupçonner Apulée d'avoir conceptualisé et prévu les atouts persuasifs de l'imprécation de la manière présentée ici. On sait toutefois que les Anciens disposaient d'un savoir-faire certain, acquis à l'école du rhéteur, et qu'ils classaient certaines figures en fonction de leurs effets sur l'auditoire ${ }^{51}$. Les manuels de rhétorique nous rappellent des figures ou 
postures comme l'optatio, la déésis, la mempsis ou encore l'exsecratio, souvent classées parmi les figures du pathos. Mais les effets escomptés, attirer la sympathie ou l'antipathie sur l'un ou l'autre personnage, laissent transparaitre un lien étroit avec l' ethos, au sens large d'image discursive de l'orateur. Les frontières entre les deux preuves techniques sont perméables, et particulièrement chez les rhéteurs latins: le conciliare cicéronien ou les réflexions de Quintilien associent les émotions modérées, comme l'amitié ou la sympathie (amor) aux mores, et donc à l'image de l'orateur, dont le moment d'élaboration privilégié est l'exorde ${ }^{52}$. Cicéron, qui figure sans doute parmi les modèles d'Apulée, a eu recours à des procédés similaires dans ses plaidoiries : Harrison (2000) cite la fin de la cinquième action contre Verrès (cicéron, In Verr. act. sec. V, 184-189), mais on trouve aussi un autre exemple d'invocation dans le Pro Milone (85-86). Même si les contextes et les affaires sont très différentes, on peut penser que Cicéron poursuivait en partie les mêmes buts qu'Apulée: il se présente comme un homme intègre et pieux, se place sous le patronage des divinités traditionnelles et demande leur aide pour réclamer de la part des juges une sentence exemplaire qui ferait office de vengeance sacrée pour les sacrilèges commis par Verrès et Clodius. Apulée a donc pu imaginer les effets d'un tel discours grâce à ses connaissances techniques.

\section{Conclusion}

En conclusion, on aurait tort de penser que l'extrait analysé de la défense d'Apulée n'aurait pour unique fonction que de livrer, par inadvertance ou impertinence, une nouvelle preuve de ses connaissances ésotériques, ou quelque recette de magie antique, et d'entretenir ainsi une réputation dont l'auteur n'est pas parvenu à se débarrasser. $\mathrm{Ce}$ serait oublier la vocation première de l'Apologie : celle d'être un discours judiciaire de défense visant à repousser une accusation de magie face à un certain auditoire dans un procès au tribunal, qu'il soit réel ou fictif. Dans les chapitres 63 et 64 , Apulée tente de rejeter sur l'accusateur un soupçon d'impiété, corollaire naturel de la magie dans les traits appartenant à la figure marginalisée du sorcier, et de se placer à nouveau dans le champ de l'acceptable, celui de la religion, de la philosophie, de la science et de la culture. Pour ce faire, il a recours à un procédé à la fois simple et efficace : l'imprécation sous la forme d'un appel à une divinité. Mais il fallait sans doute le talent et l'audace de cette personnalité singulière pour revisiter ce procédé, dans un contexte qui s'y prêtait parfaitement, et exploiter à plein rendement ses propriétés pragmatiques et sa dimension persuasive : par le biais des présupposés, l'attaque vient alimenter le renversement des postures sur le plan de l'ethos, preuve qui est un enjeu majeur dans la stratégie argumentative mise en place par Apulée; car celui-ci veut défendre sa propre image autant que ses actes. Et quand on est armé de la rhétorique, il n'est nul besoin de magie pour y parvenir et triompher de son adversaire. 


\section{BIBLIOGRAPHY}

Abt, Adam. 1967 [1908]. Die Apologie von Apuleius von Madaura und die antike Zauberei. Beiträge zur Erlauterung der Schrift de magia (Berlin : Giessen)

Albert, Luce \& Loïc Nicolas. 2010. «Le "pacte” polémique ; enjeux rhétoriques du discours de combat ", Albert, Luce \& Loïc Nicolas (éds). Polémique et rhétorique. De l'antiquité à nos jours (Bruxelles : De Boeck - Duculot), 17-48

Amossy, Ruth. 2006. L'argumentation dans le discours (Paris : Armand Colin)

Anderson, Graham. 1993. The Second Sophistic: A Cultural Phenomenon in the Roman Empire (Londres : Routledge)

Audollent, Auguste. 1903. « Devotio ou defixio », Fontemoing, Albert (éd.). Mélanges G. Boissier (Paris : Albert Fontemoing), 36-63

Austin, John Langshaw. 1962. How to Do Things with Words (Oxford : Oxford University Press). 2002 [1972]. Quand dire, c'est faire. Trad. française par G. Lane (Paris : Seuil)

Asztalos, Monika. 2005. « Apuleius' Apologia in a Nutshell : the Exordium », Classical Quarterly

$55,266-276$

Beaujeu, Jean. 1973. Apulée. Opuscules philosophiques et fragments. Texte établi et traduit par J. B. (Paris : Les Belles Lettres)

Bracops, Martine. 2006. Introduction à la pragmatique. Les théories fondatrices : actes de langage, pragmatique cognitive, pragmatique intégrée (Bruxelles : De Boeck)

Bradley, Keith. 1997. « Law, Magic, and Culture in the Apologia of Apuleius », Phoenix 51, 203-223 Butler, Harold Edgeworth \& Arthur Syngf Owen. 1967. Apulei Apologia sive pro se de magia liber. With Introduction and Commentary by H. E. B. and A. S. O. (Hildesheim : G. Olms)

Callebat, Louis. 1984. « La prose d'Apulée dans le De Magia », Wiener Studien 18, 143-167

Clément, Fabrice. 2003. « L'esprit ensorcelé. Les racines cognitives de la sorcellerie », Terrain 4, 121-136

Danblon, Emmanuelle. 2002. Rhétorique et rationalité. Essai sur l'émergence de la critique et de la persuasion (Bruxelles : Éditions de l'Université Libre de Bruxelles)

David, Jean-Michel. 1980. « Maiorum exempla sequi : l'exemplum historique dans les discours judiciaires de Cicéron », Mélanges de l'École française de Rome. Moyen-Age, Temps modernes 92, $1,67-86$

Diouf, Eugène. 2005. «L'Apologie d'Apulée : la rhétorique au secours d'un étranger. Alii orabunt causas melius (Virgile, En., 6, 849) “D'autres feront de meilleures plaidoiries" ", Lehmann, Yves, Gérard Freyburger \& James Hirstein (éds). Antiquité tardive et humanisme. De Tertullien à Beatus Rhenanus. Mélanges offerts à François Heim à l'occasion de son $70^{\mathrm{e}}$ anniversaire (Turnhout : Brepols), 92-99

Ducrot, Oswald. 1984. Le dire et le dit (Paris : Les Éditions de Minuit)

Ducrot, Oswald. 1972. Dire et ne pas dire (Paris : Les Éditions de Minuit)

Eggs, Ekkehard. 1994. Grammaire du discours argumentatif (Paris : Kimé) 
Faraone, Christopher A. 1999. «Curses and Social Control in the Law Courts of Classical Athens ", Dike 2, 99-121

Faraone, Christopher A. 1991. " The Agonistic Context of Early Greek Binding Spells », Faraone, Christopher A. \& Dirk Obbink (éds). Magika Hiera. Ancient Greek Magic and Religion (New York Oxford : Oxford University Press), 3-32

Fick, Nicole. 1992. « Mariage d'argent, mariage d'amour : la magie du mariage d'Apulée », Vita Latina 125, 129-146

Fick, Nicole. 1991. « Magie et religion dans l'Apologie d'Apulée », Vita Latina 124, 14-46

Gaide, Françoise. 1993. « Apulée de Madaure a-t-il prononcé le De Magia devant le proconsul d'Afrique », Les Études Classiques 61, 227-231

Gleason, Maud W. 1995. Making Men. Sophists and Self-presentation in Ancient Rome (Princeton, New Jersey : Princeton University Press)

Gordon, Richard. 1999. « Imagining Greek and Roman Magic », Ankarloo, Bengt \& Stuart Clark (éds). Witchcraft and Magic in Europe, 2 : Ancient Greece and Rome (Philadelphie : University of Pennsylvania Press), 161-275

Goyet, Francis. 2013. « Sur la nécessaire collaboration des latinistes pour la recherche en rhétorique ", Cahiers des études anciennes [En ligne : http://etudesanciennes.revues.org/594, consulté le 11 juillet 2014]

Goyet, Francis. 1996. Le sublime du « lieu commun ", L'invention rhétorique dans l'Antiquité et à la Renaissance (Paris : Champion)

Graf, Fritz. 1994. La magie dans l'Antiquité gréco-romaine (Paris : Les Belles Lettres)

Guey, Julien. 1951. « Au théâtre de Leptis Magna. Le proconsulat de Lollianus Avitus et la date de l'Apologie d'Apulée », Revue des Études Latines 29, 307-317

Habermehl, Peter. 2002. « Magie, Mächte und Mysterien : Die Welt des Übersinnlichen im Werk des Apuleius », Hammerstaedt, Jürgen, Peter Habermehl, Francesca Lamberti, Adolf Ritter \& Peter Schenk. Apuleius. De Magia. Eingeleitet, übersetzt und mit interpretierenden Essays versehen von J. H., P. H., Fr. L., A. R. \& P. S. (Darmstadt : Wissenschaftliche Buchgesellschaft), 285-314.

Halsall, Albert W. 1995. Victor Hugo et l'art de convaincre. Le récit hugolien : rhétorique, argumentation, persuasion (Montréal : Les Éditions Balzac)

Halsall, Albert W. 1988. L'art de convaincre. Le récit pragmatique, rhétorique, idéologie, propagande (Toronto : Paratexte)

Hammerstaedt, Jürgen, Peter Habermehl, Francesca Lamberti, Adolf Ritter \& Peter Schenk. Apuleius. De Magia. Eingeleitet, übersetzt und mit interpretierenden Essays versehen von J. H., P. H., Fr. L., A. R. \& P. S. (Darmstadt : Wissenschaftliche Buchgesellschaft)

Harrisson, Stephen. 2008. «The Sophist At Play in Court : Apuleius'Apology and his Literary Career », Werner, Riess (éd.). Paideia at Play : Learning and Wit in Apuleius (Groningen : Barkhuis Publishing \& Groningen University Library), 3-15

Harrison, Stephen. 2000. Apuleius. A Latin Sophist (Oxford : Oxford University Press)

Helm, Rudolf. 1955. « Apuleius Apologie : ein Meisterwerk der Zweiten Sophistik », Altertum 1, 86-108 
Hertz, Géraldine. 2010. «Apulée contre Émilianus dans l'Apologie : art de la polémique et cas problématique de la malédiction de l'adversaire ", Albert, Luce \& Loïc Nicolas (éds). Polémique et rhétorique. De l'Antiquité à nos jours (Bruxelles : De Boeck - Duculot), 105-118.

Hijmans, Benjamin Lodewijk. 1994. « Apuleius Orator : 'Pro se de Magia' and 'Florida' »; Aufstieg und Niedergang der Römischen Welt 2, 34, 2, 1709-1784

Hijmans, Benjamin Lodewijk. 1987. « Apuleius Philosophicus Platonicus », Aufstieg und Niedergang der Römischen Welt 2, 36, 1, 395-475

Hunink, Vincent. 2008. « Homer in Apuleius'Apology », Werner, Riess (éd.). Paideia at Play : Learning and Wit in Apuleius (Groningen : Barkhuis Publishing \& Groningen University Library), 75-87

Hunink, Vincent. 2001. « Introduction ». Harrison, stephen, John Hilton \& Vincent Hunink (éds). 2001. Apuleius. Rhetorical Works (Oxford : Oxford University Press), 11-24

Hunink, Vincent. 1997. Apuleius of Madauros Pro se de Magia, 2 vol. Edited with a Commentary by V. H. (Amsterdam : J. C. Gieben)

Kerneis, Soazick. 2013. « Des justices "populaires" dans l'Empire romain (II ${ }^{\mathrm{e}}-\mathrm{IV}^{\mathrm{e}}$ siècles) », Droit et Cultures 65, 89-99

Lamberti, Francesca. 2002. « De magia als rechtsgeschichtliches Dokument », Hammerstaedt, Jürgen, Peter Habermehl, Francesca Lamberti, Adolf Ritter \& Peter Schenk. Apuleius. De Magia. Eingeleitet, übersetzt und mit interpretierenden Essays versehen von J. H., P. H., Fr. L., A. R. \& P. S. (Darmstadt : Wissenschaftliche Buchgesellschaft), 331-350

Lausberg, Heinrich. 1960. Handbuch der literarischen Rhetorik. Eine Grundlegung der Literaturwissenschaft, 2 vol. (Munich : Max Hueber Verlag) Leumann, Manu, Johann B. Hofmann \& Anton Szantyr. 1977 [1926-1928]. Lateinische Grammatik. Erster Band. Lateinische Laut- und Formen-Lehre (Munich : C. H. Beck)

Núñez, Loreto. 2009. « Apuleius orator metasophisticus. Miroirs d'un orateur », van Mal-Maeder, Danielle, Alexandre Burnier, Loreto Núñez (éds). Jeux de voix. Enonciation, intertextualité et intentionnalité dans la littérature antique (Berne : Peter Lang)

Maingueneau, Dominique. 2010. Manuel de linguistique pour les textes littéraires (Paris : Armand Colin)

Martin, Michaël. 2010. Sois Maudit ! Malédictions et envoûtements dans l'Antiquité (Paris : Errance)

Mauss, Marcel. 1973. Sociologie et anthropologie (Paris : PUF)

McCreight, Thomas D. 2008. « The "Riches" of Poverty : Literary Games with Poetry in Apuleius' Laus Paupertatis (Apology 18) », Riess, Werner (éd.). Paideia at Play : Learning and Wit in Apuleius (Groningen : Barkhuis Publishing \& Groningen University Library), 89-104

McCreight, Thomas D. 1991. Rhetorical Strategies and Word Choice in Apuleius' Apology (Durham, NC : Diss. Duke)

McCreight, Thomas D. 1990. « Invective Techniques in Apuleius’Apology », H. Hofmann (éd.). Groningen Colloquia on the Novel 3 (Groningen : Egbert Forsten), 35-62

Méthy, Nicole. 2000. « Magie, religion et philosophie au second siècle de notre ère. À propos du Dieu-roi d'Apulée », Moreau, Alain \& Jean-Claude Turpin (éds). La Magie. Actes du colloque international de Montpellier (Montpellier : Université Montpellier III), 85-107 
Micheli, Raphaël. 2010. L'émotion argumentée. L'abolition de la peine de mort dans le débat parlementaire français (Paris : Cerf)

Mortley, Raoul. 1972. « Apuleius and Platonic Theology », American Journal of Philology 93, $584-590$

Patillon, Michel \& Giancarlo Bolognesi. 2002 [1997]. Aelius Theon. Progymnasmata. Texte établi et traduit par P. M. avec l'assistance, pour l'arménien, de G. B. (Paris : Les Belles Lettres)

Perelman, Chaïm \& Lucie Olbrechts-Tyteca. 2008 [1958]. Traité de l'argumentation (Bruxelles : Les Éditions de l'Université Libre de Bruxelles)

Puccini-Delbey, Géraldine. 2010. « Apulée, un nouveau Socrate ? : une analyse des rapports d'intertextualité entre le “ De magia " et "l'Apologie” de Platon », Latomus 69, 429-445

Puccini-Delbey, Géraldine. 2004. De Magia d'Apulée (Neuilly : Atlande)

Orlandini, Anna. 1999. « De la connexion : une analyse pragmatique des connecteurs latins autem et ceterum », Indogermanische Forschnungen 104, 142-163

Ortony, Andrew, Gerald L. Clore \& Allan Collins. 1988. The Cognitive Structure of Emotions (Cambridge : Cambridge University Press)

Pernot, Laurent. 2000. La Rhétorique dans l'Antiquité (Paris : Le livre de poche)

Riemer, Ulrike. 2006. « Apuleius, De magia. Zur Historizität der Rede », Historia 55, 178-190

Riess, Werner. 2008. " Apuleius Socrates Africanus ? Apuleius' Defensive Play », Werner, Riess (éd.). Paideia at Play : Learning and Wit in Apuleius (Groningen : Barkhuis Publishing \& Groningen University Library), 51-73

Rives, James B. 2006. « Magic, Religion, and Law : the Case of Lex Cornelia de sicariis et veneficis ", Ando, Clifford \& Jörg Rüpke (éds). Religion and Law in Classical and Christian Rome (Stuttgart : Franz Steiner), 47-67

Rives, James B. 2008. « Legal Strategy and Learned Display in Apuleius'Apology », Werner, Riess (éd.). Paideia at Play : Learning and Wit in Apuleius (Groningen : Barkhuis Publishing \& Groningen University Library), 17-49

Sallmann, Klaus 1995. «Erzählendes in der Apologie des Apuleius, oder : Argumentation als Unterhaltung ", H. Hofmann (éd.). Groningen Colloquia on the Novel 5 (Groningen : Egbert Forsten), 137-158

Sandy, Gerald. 1997. The Greek World of Apuleius. Apuleius and the Second Sophistic (LondresNew York-Cologne : Brill)

Schenk, Peter. 2002. « Einleitung », Hammerstaedt, Jürgen, Peter Habermehl, Francesca Lamberti, Adolf Ritter \& Peter Schenk. Apuleius. De Magia. Eingeleitet, übersetzt und mit interpretierenden Essays versehen von J. H., P. H., Fr. L., A. R. \& P. S. (Darmstadt : Wissenschaftliche Buchgesellschaft), 23-57

Schindel, Ulrich. 1996. « Die Verteidigungsrede des Apuleius », Ulrich Mölk (éd.). Literatur und Recht. Literarische Rechtsfälle von der Antike bis in die Gegenwart (Göttingen : Wallstein), 13-24

Schindel, Ulrich. 2000. « Apuleius - Africanus Socrates? Beobachtungen zu den Verteitigungsreden des Apuleius und des platonischen Socrates », Hermes 128, 443-456 Schneider, Catherine. 2008. « Discours écoutés, discours prononcés dans l'Afrique romaine l'Apologie d'Apulée ou le trompe-l'œil absolu », Abbamonte, Giancarlo, Lorenzo Miletti \& Luigi Spina (éds), Discorsi alla prova. Atti del Quinto Colloquio italo-francese. Discorsi pronunciati, 
discorsi ascoltati : contesti di eloquenza tra Grecia, Roma ed Europa, Napoli - S. Maria di Castellabate (Sa) 21 - 23 settembre 2006 (Naples : Giannini Editore), 391-419

Searle, John. 1982. Sens et expression. Études de théorie des actes de langage. Traduction et préface par J. Proust (Paris : Les Éditions de Minuit)

Stock, Fabio. 1985. « Il pauperismo di Apuleio », Index 13, 353-386

Tilg, Stephan. 2008. «Eloquentia ludens - Apuleius' Apology and the Cheerful Side of Standing Trial », Werner, Riess (éd.). Paideia at Play : Learning and Wit in Apuleius (Groningen : Barkhuis Publishing \& Groningen University Library), 105-132

Valette, Paul. 1924. Apulée. Apologie. Florides. Texte établi et traduit par P. V. (Paris : Les Belles Lettres)

Valette, Paul. 1908. L'apologie d'Apulée (Paris : Klincksieck)

Vanderveken, Daniel. 1988. Les actes de discours. Essai de philosophie du langage et de l'esprit sur la signification des énonciations (Liège-Bruxelles : Pierre Mardaga)

Versnel, Henk S. 1991. «Beyond Cursing: The Appeal to Justice in Judicial Prayers », Faraone, Christopher A. \& Dirk Obbink (éds). Magika Hiera. Ancient Greek Magic and Religion (New York Oxford : Oxford University Press), 60-106

Versnel, Henk S. 1987. « Les imprécations et le droit », Revue d'Histoire du Droit Français et Étranger 65, 5-22

Walker, Jeffrey. 2000. Rhetoric and Poetics in Antiquity (Oxford : Oxford University Press)

Webb, Ruth. 1997. «Mémoire et imagination : les limites de l'enargeia dans la théorie rhétorique grecque ", Lévy, Carlos \& Laurent Pernot. Dire l'Évidence. Philosophie et rhétorique antiques (Paris : L'Harmattan), 229-248

Webb, Ruth. 2012 [2009]. Ekphrasis, Imagination and Persuasion in Ancient Rhetorical Theory and Practice (Farnham - Burlington : Ashgate)

Whitehead, David. 1979. « Tacitus and the Loaded Alternative », Latomus 38, 474-495

Winter, Thomas Nelson. 1969. « The Publication of Apuleius Apology », Transactions and Proceedings of the American Philological Association 100, 107-112

Winter, Thomas Nelson. 1968. Apology as Prosecution. The Trial of Apuleius (Evanston, Illinois : Northwestern University)

Wisse, Jakob. 1989. Ethos and Pathos from Aristotle to Cicero (Amsterdam : Hakkert)

Youni, Maria S. 2012. "L'imprécation et la loi : châtiment divin et sanctions pénales dans la polis grecque », Helmis, Andréas, Nathalie Kálnoky et Soazick Kerneis (éds), Vertiges du droit. Mélanges franco-helléniques à la mémoire de Jacques Phytilis (Paris : L’Harmattan), 399-410

\section{NOTES}

1. Sur la date probable du procès d'Apulée, voir Schenk 2002 : 13-16; Hunink 1994 : 1712-1714. L'hypothèse de Guey (1951 : 307-317), selon laquelle Maximus aurait été proconsul en 158/9 de notre ère, est désormais la plus largement acceptée.

2. Dans cette action contre Apulée, Aemilianus s'est allié à un certain Herennius Rufinus, qui aurait donné sa fille en mariageà Pontianus. 
3. La mésaventure d'Apulée rappelle les attaques de Polémon à l'encontre de Favorinus d'Arles (Gleason $1995: 8$ ).

4. L'interprétation de Graf est parfaitement compatible avec les réflexions des psychologues cognitivistes sur le succès transculturel de la magie (voir notamment Clément 2003 : 121-136).

5. L'hypothèse la plus largement acceptée est que les maléfices tombent sous le coup de la lex Cornelia de sicariis et ueneficiis, qui remonte à Sylla (Puccini-Delbey $2004: 28$; Harrison $2000: 41$; Gordon 1999 : 263 ; Hunink $1997: 12-14$; $2001: 12$; Graf $1994: 71 ; 80)$. Elle a récemment été contestée par Lamberti (2002) et Rives (2008: 19-25; 2006).

6. Apol. 1, 3: Quo ego uno praecipue confisus gratulor medius fidius, quod mihi copia et facultas te iudice obtigit pugandae apud imperitos philosophiae et probandi mei. Cette stratégie rappelle celle de Cicéron dans le Pro Archia ou celle de l'Apologie de Socrate de Platon (Riess 2008 : 53 ; 56 ; McCreight 1990 : 43).

7. Sur l'organisation et la composition du discours d'Apulée, voir Harrison $2008: 12$; $2000: 47-50$ ; Asztalos 2005 ; Schenk 2002 : 26-30 ; Hunink 2001 : 14-21 ; Hijmans 1994 : 1724-1726.

8. Sur Mercure et l'appellation «basileus» en contexte magique, voir Habermehl $2002: 288-289$; Sandy 1997 : 25 ; Graf 1994 : 97-100 ; Fick 1991 : 23-27 ; Regen 1971 : 94-103 ; Winter 1968 : 81-85 ; 100-106; Abt 1967 [1908] : 299-303.

9. Graf 1994 : 100-105 ; Fick 1991 : 14-31. Apulée témoigne lui-même de cette division (Apol. 25, 9 26 , 9), mais celle-ci est avant tout sociale et culturelle (cf. Méthy $2000: 99$ ).

10. Texte de P. Valette (CUF, 1924) ; traduction personnelle.

11. Rappelons ici que la défense d'Apulée ne tend pas à nier l'existence de la magie. Le philosophe de Madaure reconnaît même une certaine forme de magie, liée à la religion, comme digne d'estime et d'intérêt (Apol. 25, 9-27,4). Ce qu'il nie, c'est d'avoir utilisé une forme de magie malveillante contre autrui.

12. Gaide 1993 : 230 : « une dangereuse parodie de defixio »; Hertz $2010: 112$ : « le magus perce soudain dangereusement sous le masque du philosophe»; 113: "L'attaque ici dessert étrangement son auteur, car il se rapproche de la défintion du magus qu'il donnait auparavant (Apol. 26)»).

13. Nous nous joignons à l'exhortation de Francis Goyet (2013) et à l'idée ancienne que l'acquisition et la compréhension de la technique rhétorique associe étroitement la théorie et la pratique, cette dernière comprenant des exercices de production et d'analyse de discours.

14. Butler et Owen $1967: 128$. Pour un commentaire morphologique, voir Leumann, Hofmann et Szantyr, 1977 [1926-28] : 528.

15. Cette hyptohèse est notamment formulée par Abt (1967 [1908] : 300-303), suivi par Regen (1971: 94). Pour les différents éléments que j'ai énumérés et qui tendent à autoriser le rapprochement entre le texte et la defixio, voir Martin 2010 : 9-11 ; 29-31 ; Hunink 1997 : 169 ; Graf 1994 : $141 ; 147$; 176-185 ; 246 ; Faraone 1991 : 3-32.

16. La force illocutoire désigne l'intensité avec laquelle le but d'un acte de langage est mis en œuvre ou recherché. C'est ce qui distingue l'ordre de la simple requête. Sur cette notion, voir Searle 1982 : 44 ou Bracops $2006: 49 ; 202$. Vanderveken $(1988: 113-115 ; 121-123)$ parle quant à lui du mode d'accomplissement ou du degré de puissance.

17. Cela permet peut-être de rapprocher les paroles d'Apulée d'autres formes de magie, publiques et plus conventionnelles, comme les imprécations funéraires ou les prières pour la justice (voir Kerneis 2013 : 89-99 ; Martin 2012 : 76-78 ; 83-85 ; Versnel 1987 : 5-22 ; 1991 : 60-106 ; Youni 2012 : 399-410).

18. La version du Pro Milone que nous avons conservée a été réécrite ; l'actio secunda in Verrem constitue un exemple fameux de discours qui n'a pas été effectivement prononcé.

19. Sur l'authenticité et l'éventuel remaniement du discours d'Apulée, voir Schneider 2009 : 391-395 ; Harrison $2008: 14$; $2000: 42$; 75, n. 93 ; Hunink 2008 : 75-76, n. 3 ; 86-87, n. 32 ; 2001 : 21-24 ; 1997: 21 ; 25-27 ; McCreight 2008 : 90, n. 9 ; Rives 2008 : 17-19 ; Riemer 2006 : 178-190 ; 
Schenk 2002 : 39-43 ; Walker 2000 : 121 ; Sandy $1997: 131$; Hijmans $1994: 1715-1719$; Gaide 1993 : 227-231; Callebat 1984 : 143, n. 1 ; Abt 1967 [1908] : 76-88 ; Valette 1924 : XXIV ; 1908 : 118.

20. La plupart des arguments cités ont été déjà - sauf pour les plus anciens - repoussés par Winter (1969 : 107-112 ; 1968 : 25-31. Rives (2008) situe quant à lui le discours d'Apulée dans le cadre lâche d'une cognitio extra ordinem portant sur le mariage avec Pudentilla, mais aussi sur l'accusation plus vague et difficile à repousser d'être un magus. Dans cette hypothèse, les développements précédant la question du mariage et apparemment sans lien avec l'affaire, et les nombreuses démonstrations d'érudition et d'éloquence, parce qu'elles sont la marque d'une élite sociale, sont essentiels dans la stratégie de défense d'Apulée.

21. Selon Callebat (1984: 143, n. 1), suivi par Bradley (1997: 213, n. 19), le texte a été revu pour des corrections mineures, mais n'a pas été pas recomposé ou réécrit ; Abt (1967 [1908] : 76-88), Harrison (2000: 42) et Walker (2000: 121) estiment que le texte a été considérablement retravaillé ; pour Gaide (1993 : 231), seule la dernière partie du discours est authentique. Cette section comporte en effet de nombreux détails matériels (usage de l'argent de Pudentilla, lecture des actes, testaments, achats immobiliers) qui sont en fort contraste avec les amplifications qui précèdent.

22. Sur l'aspect déclamatoire du discours d'Apulée, voir : Riess 2008 : 51-73 ; Schenk 2002 : 46-56 ; Harrison 2000: 86-88; Walker 2000: 126-127 ; Sandy 1997: 131-148 ; Hijmans 1994: 1711 ; McCreight 1990. Catherine Schneider (2009) montre en particulier qu'à l'exception de la fable, l' Apologie reprend tous les types d'exercices préparatoires de rhétorique (progymnasmata) de façon démonstrative et didactique. Le discours se donne dès lors comme une sorte de manuel d'exercices appliqués. Cet aspect (qui fait partie de la construction de l'image de soi et de la stratégie de défense, voir Rives 2008 ; Tilg 2008) mis à part, on peut comprendre que, dans l'hypothèse d'un procès réel et tendu, Apulée se soit appuyé sur les formes qu'il maîtrise et qui visaient précisément à nourrir un discours complet (Patillon \& Bolognesi 2002 [1997] : XVIII).

23. Même si elle est l'un des seuls témoignanges direct d'éloquence judiciaire pour cette époque (McCreight 2008: 92 ; Hunink 1997 : 23 ; Sandy 1997: 145-146), l'Apologie d'Apulée est généralement considérée comme une des oeuvres majeures de ce mouvement culturel et intellectuel (Harrisson 2000 ; Sandy 1997 ; Anderson 1993 : 223-227 ; Helm 1955).

24. Apulée fait notamment passer la citation d'un traité d'ichtyologie en langue grecque pour des formules magiques (Apol. 38, 7-9) et énumère des noms de magiciens (Apol. 91, 1).

25. Citons par exemple, Cicéron, In Pis., 68-71 ; Pro Mil., 72-73; Quintilien Inst. 2, 1, 11. Sur l'insulte extra causam et le discours d'Apulée, voir Tilg 2008 : 106-107 ; McCreight 1990 : 35-62. L'athéisme fait partie des attaques les plus courantes.

26. Il en tire même argument au chapitre 26 : un magicien, affirme-t-il, aurait pu échapper au procès ou lancer les pires représailles contre ses détracteurs; ces derniers auraient commis une erreur fatale en accusant quelqu'un qui détient de tels pouvoirs, contre lesquels ils ne peuvent se prémunir ; ils ne croient donc pas qu'Apulée soit réellement capable de leur jeter un sort.

27. Habermehl $2002: 289$; Harrison $2000: 74-75:$ «a splendidly melodramatic curse (...) the quasi-magic colouring of the curse suggests that Apuleius is playing up to the magic powers attributed to him ». La capacité à se constituer ou à jouer des personae est un trait prégnant de la seconde sophistique (voir par exemple Núñez 2009 ; Riess 2008 ; Gleason 1995).

28. Le Corax est un procédé qui consiste à dire qu'il n'est pas vraisemblable qu'une personne soit coupable de ce qu'on lui reproche, précisément parce qu'elle n'ignore pas que toutes les vraisemblances sont contre elle (Aristote, Rhét. II, 24, 1402a ; Perelman et Olbrechts-Tyteca, 2008[1958] : 608-609). Cette technique rhétorique est notamment illustrée par la défense de la romancière Catherine Tramell dans le sulfureux Basic Instinct.

29. Pour une brève histoire de la rhétorique dans l'Antiquité, voir Pernot 2000.

30. La rupture de liaison consiste à soutenir que deux éléments sont indûment associés (Perelman et Olbrechts-Tyteca, 1958 [2008] : 550-551). 
31. En effet, l'exclamation présuppose l'affirmation du contenu propositionnel (Cf. Danblon 2002 :136).

32. Liée à l'enargeia, l'ekphrasis est un mode particulier de description censé provoquer un effet de perception qui ne se limite pas à la dimension matérielle de l'objet, mais touche aussi à l'imagination et aux émotions (Danblon 2002 : 186 ; Webb 1997 : p. 229-248 ; 2012 [2009]).

33. La forme laruans serait l'exemple unique d'une recréation d'un verbe laruare (OLD, laruare, p. 1003-1004), sur la base de la forme laruatus, qui n'est attestée que six fois dans toute la littérature latine. Certaines traductions (Valette, CUF, $1924: 77$ : «Prendre cela pour un spectre des Enfers, c'est être soi le jouet des spectres infernaux»; Hunink $2001: 86$ : "whoever thinks this is a ghost is clearly haunted himself») donnent à laruans un sens quasi identique à celui de laruatus, mais la forme de participe présent, de voix active, correspond peut-être mieux à la rétorsion que tente effectuer l'orateur. Le texte signifie qu'Aemilianus veut ensorceler, et non pas qu'il est victime d'un sort (cf. Hammersteadt 2002: 169: "wer diesen schließlich für ein Gespenst hält, beschwört selbst Gespenster herauf. »).

34. Dans son long discours, Apulée a tendance à renouveler ses attaques. On trouve ainsi des tentatives pour discréditer l'adversaire sur le plan religieux en Apol. 26-27 (en particulier, 27, 10) et en 56, 3-10.

35. Sur cette partie de la défense d'Apulée, voir en particulier Méthy (2000).

36. On a depuis longtemps observé les liens entre le discours d'Apulée, qui se considérait luimême comme un Socrates Africanus, et les apologies de Socrate (Puccini-Delbey 2010 ; Núñez 2009: 289; Riess 2008; Harrisson 2000: 43; 51; 96; Schindel 2000; 1996; Helm 1955 : 101). Rappelons à titre d'exemple le début des Mémorables de Xénophon (I, 1, 2 et suivants). La structure argumentative graduelle est la même que celle qui organise les chapitres 63-64 de l' Apologie: Xénophon montre que Socrate respectait les dieux de la cité, que ses pratiques n'avaient rien d'anormal, mais aussi que grâce à son démon, et au lien particulier qu'il avait avec la divinité, il était mieux placé que les autres pour comprendre la volonté des dieux et aider son prochain par ses conseils inspirés.

37. Sur ce silence et le platonisme d'Apulée, voir entre autres Tilg $2008: 121$; Sandy $1997: 22-26$ ; Hunink 1987 : 416-425 ; Mortley 1972.

38. Sur les catégories « dite », « montrée » et « suscitée » de l'émotion, voir Micheli (2010).

39. Cf. Aristote, Rhét. II, 9, 1386b. J'utilise ici le modèle de R. Micheli (L'émotion argumentée, 2010) qui s'inspire à la fois de la manière dont Aristote décrit les émotions dans le deuxième livre de la Rhétorique et de la théorie de l'appraisal en psychologie cognitive (cf. Ortony, Clore et Collins 1988) selon laquelle chaque émotion comporte une évaluation, rationnelle mais subjective, par le sujet de la situation face à laquelle il se trouve. Les paramètres de cette évaluation (survenue probable d'événements considérés comme négatifs ou positifs, conformité aux valeurs ou à l'image de soi) sont susceptibles d'être exploités au niveau discursif et de constituer la base d'une topique des émotions.

40. Cf. Aristote, Rhét. II,5, 1382a. Voir aussi Micheli $2010: 271-285$.

41. Habermehl $2002: 289$; Abt 1967 [1908] : 230-231. Hunink semble être du même avis (1997: 169 : « vehement, threatening curse of Aemilianus »).

42. La possibilité plus ou moins grande qu'un événement désirable ou indésirable se produise, mais aussi la capacité de maîtrise de la situation, figurent parmi les critères de l'évaluation de la situation par le sujet qui guide l'émotion.

43. Typique de cette éducation est la capacité à faire des citations et à fournir des listes (Rives 2008: 40-43) d'auteurs ou de termes d'un même domaine, ce que le passage discuté pourrait également illustrer (lemurum, manium, laruarum ; occursacula, formidamina, terriculamenta).

44. La manière dont Apulée pratique le culte paraît très singulière au regard des formes traditionnelles de la religion antique et avait peut-être de quoi soulever l'étonnement ou le 
soupçon (Graf 1994 : 96-100). Mais ce qui m'importe ici est la façon dont l'orateur veut présenter son action.

45. Sur les emplois de ceterum, voir Orlandini 1999 : 142-163.

46. Searle (1982) parle d'« état psychologique»; Vanderveken (1988) utilise les paramètres de "condition de sincérité », de "condition préparatoire» et de "condition sur le contenu propositionnel ». Il ne me paraît pas indispensable de déterminer à quelle catégorie précise d'acte de langage appartient l'imprécation d'Apulée: il s'agit vraisemblablement d'un acte ambigu ou complexe, mélangeant la force directive (demande) et déclarative (à laquelle appartient le verbe « maudire » en français selon Vanderveken). Les critères d'évaluation et de classification suffisent pour mon propos : il faut que l'auteur jouisse de l'autorité nécessaire pour faire la demande, qu'il désire que l'action demandée soit accomplie, qu'il croie que son allocutaire est capable d'accomplir cette action, que celle-ci soit future, etc.

47. Sur cette notion, voir Ducrot 1984 ; 1972. Pour un résumé efficace de la théorie ducrotienne, voir Bracops 2006 : 150-158.

48. Une phrase du type "J'affirme que $P$ mais je ne crois pas que $P$ » paraît difficilement envisageable. C'est dès lors que l'affirmation implique la croyance que le contenu propositionnel est vrai (Searle1982: 43 ; 52). Un autre exemple est qu'à la déclaration "je vous bénis ", la réponse «mais vous n'êtes pas prêtre » constitue une objection pertinente. Celui qui accomplit un acte de langage, du simple fait qu'il énonce, postule généralement que les conditions de réussite de cet acte sont réunies (cf. Maingueneau 2012 : 22-23).

49. Apulée, Démon, 15, 152-153 (texte de J. Beaujeu, CUF, 1973) : Est et secundo significatu species daemonum animus humanus emeritis stipendiis uitae corpore suo abiurans. Hunc uetere Latina lingua reperio Lemurem dictitatum. Ex hisce ergo Lemuribus qui posterorum suorum curam sortitus placato et quieto numine domum possidet, Lar dicitur familiaris; qui uero ob aduersa uitae merita nullis [bonis] sedibus incerta uagatione ceu quodam exilio punitur, inane terriculamentum bonis hominibus, ceterum malis noxium, id genus plerique Laruas perhibent. Cum uero incertum est, quae cuique eorum sortitio euenerit, utrum Lar sit an Larua, nomine Manem deum nuncupant (...). Ce passage, souvent cité pour éclairer le paragraphe 64, 1 de l'Apologie (Cf. Hunink 1997: 164 ; Butler et Owen 1967 : 128 ; Valette $1924: 77$, n. 1) laisse penser que si Apulée trouve dans la longue énumération des créatures infernales une nouvelle occasion d'étaler sa science, ici démonologique, il fait aussi appel à ce qu'il considère comme un fonds traditionnel de la langue et de la spiritualité romaine (uetere Latina lingua).

50. Dans ses discours, Cicéron rappelle souvent les actions d'ancêtres illustres pour contraindre les juges à prendre une décision conforme à une certaine ligne politique, aux valeurs et aux traditions romaines (voir David $1980: 67-86$ ).

51. Lausberg 1960 : 336-344. Pour une application pratique de ces figures, voir les travaux de A. W. Halsall consacrés au roman réaliste (Halsall 1988 : 214-236 ; 1995 : 321-346).

52. Cicéron, De Or. II, 182 ; Quintilien, Inst. VI, 2, 8-9. Voir à ce sujet, Wisse 1989 : 233-245 ; Goyet 1996 : 261-267 ; 279-301.

\section{ABSTRACTS}

In this paper, I discuss a controversial extract of Apuleius' Apology $(64,1)$. This passage, where Apuleius seems to curse his adversary, has sometimes been interpreted as a blunder of the 
author, revealing his knowledge about magic. I try to argue that it actually contains a rhetorical and performative trick which perfectly fits with the global strategy of the speech.

À travers l'analyse d'un extrait controversé de l'Apologie d'Apulée $(64,1)$, l'article montre comment la rhétorique ancienne et la pragmatique contemporaine peuvent contribuer à la compréhension de passages problématiques et à l'étude de la technique déployée par l'orateur. Le passage discuté, où Apulée semble jeter une malédiction sur son adversaire, a parfois été interprété comme une imprudence de l'auteur, trahissant ses connaissances en matière de magie. L'article défend qu'il comporte en réalité un procédé rhétorique et performatif qui s'inscrit parfaitement dans la stratégie globale du discours.

INDEX

Mots-clés: apologie, Apulée, magie, pragmatique, rhétorique et philologie

Keywords: apology, Apuleius, magic, pragmatics, rhetoric and philology

\section{AUTHOR}

\section{BENOÎT SANS}

Université Libre de Bruxelles - GRAL 